\title{
A dosimetric study on radiation-induced hypothyroidism following intensity-modulated radiotherapy in patients with nasopharyngeal carcinoma
}

\author{
YUMEI XU ${ }^{1,2}$, ZHIYING SHAO ${ }^{3}$, TIANYOU TANG ${ }^{1}$, GUIHONG LIU ${ }^{1}$, \\ YUANHU YAO $^{1}$, JIANSHE WANG ${ }^{1}$ and LONGZHEN ZHANG ${ }^{1}$ \\ ${ }^{1}$ Department of Radiation Oncology, The Affiliated Hospital of Xuzhou Medical University; \\ ${ }^{2}$ Clinical College of Xuzhou Medical University; ${ }^{3}$ Department of Oncology, \\ The Affiliated Hospital of Xuzhou Medical University, Xuzhou, Jiangsu 221006, P.R. China
}

Received November 22, 2017; Accepted July 17, 2018

DOI: $10.3892 / \mathrm{ol} .2018 .9332$

\begin{abstract}
The objective of the present study was to investigate the association between thyroid gland-dosimetric parameters and hypothyroidism induced by intensity-modulated radiotherapy in patients with nasopharyngeal carcinoma (NPC). A total of 52 patients with NPC treated in the Department of Radiation Oncology of The Affiliated Hospital of Xuzhou Medical University, from May 2008 to December 2016 were retrospectively enrolled in the present study and divided into two groups based on thyroid function: The euthyroid and hypothyroid groups. The association between hypothyroidism and clinical or dosimetric parameters were analyzed. Females had a significantly increased probability of suffering from radiation-induced hypothyroidism (RIHT), compared with males $(\mathrm{P}=0.010)$. The occurrence of RIHT was significantly negatively associated with thyroid volume prior to radiotherapy $(\mathrm{P}=0.048)$. Furthermore, the mean dose (Dmean) and V50 in the hypothyroidism group were significantly increased, compared with the euthyroidism group $(\mathrm{P}=0.017$ and $\mathrm{P}=0.023$, respectively). During the treatment optimization period, dose constraints associated with the thyroid gland demonstrated a significantly protective effect on thyroid function compared with the unconstrained group ( $\mathrm{P}=0.034)$. According to the
\end{abstract}

Correspondence to: Dr Jianshe Wang, Department of Radiation Oncology, The Affiliated Hospital of Xuzhou Medical University, 99 West Huaihai Road, Xuzhou, Jiangsu 221006, P.R. China

E-mail:wjsemail118@163.com

Abbreviations: $\quad \mathrm{FT}_{3}$, free triiodothyronine; $\mathrm{FT}_{4}$, free tetraiodothyronine; IMRT, intensity-modulated radiotherapy; NPC, nasopharyngeal carcinoma; PTV, planning target volume; QoL, quality of life; RIHT, radiation-induced hypothyroidism; ROC, receiver operating characteristic; $\mathrm{TSH}$, thyroid stimulating hormone

Key words: nasopharyngeal carcinoma, intensity-modulated radiotherapy, hypothyroidism, dose-volume parameters, dose threshold receiver operating characteristic curves, the threshold value was 5,160 cGy for Dmean and 54.5\% for V50. The 3-year cumulative incidence of RIHT was $67.8 \%$ when the Dmean value was $>5,160$ cGy and $44.6 \%$ when the Dmean was $<5,160$ cGy (log rank test, $\mathrm{P}=0.036$ ). Furthermore, the 3 -year cumulative incidence was $66.1 \%$ when the V50 was $>54.5 \%$, and $29.9 \%$ when the V50 was $<54.5 \%$ (log rank test, $\mathrm{P}=0.025$ ). In conclusion, RIHT is associated with radiation dose, particularly with Dmean and V50 of the thyroid gland. Dose constraints associated with the thyroid gland significantly reduced the incidence of hypothyroidism compared with the unconstrained group.

\section{Introduction}

Radiotherapy is the primary treatment approach for patients with nasopharyngeal carcinoma (NPC) (1), during which at least a portion of the thyroid gland is exposed to radiation. Previous studies reported that the incidence of radiation-induced hypothyroidism (RIHT) was significantly increased in radiotherapy-treated patients treated with NPC, compared with those non-irradiated patients (9-53 and 3-8\%, respectively) $(2,3)$. The prognosis of patients with NPC is primarily good, where the 5 -year overall survival rate is $70-80 \%$ for stage I patients $(4,5)$. Furthermore, a complication encountered by patients with NPC is RIHT. Hypothyroidism may cause a range of clinical symptoms, including chills, fatigue and hypomnesia. Additionally, these patients may also experience hematological changes, including hyperlipidemia, coagulation disorders and abnormal levels of markers associated with cardiovascular diseases (6). All of these changes increase the risk of morbidity and mortality rates of cardiovascular diseases (7), and notably affect the quality of life $(\mathrm{QoL})$ of the patients; therefore, RIHT has attracted the attention of radiation oncologists and endocrinologists. However, the association between dose-volume parameters and the occurrence of hypothyroidism remains poorly understood. Furthermore, follow-up procedures to ensure normal thyroid function following radiotherapy have not been widely adopted. To avoid potential confounding effects from the use of surgery for the treatment of head and neck cancer types, patients with 
NPC were selected to investigate the factors that affect RIHT following intensity-modulated radiotherapy (IMRT), including radiation dosage and volume. The present study aimed to identify an effective way to reduce the incidence of RIHT in and improve the QoL of patients with NPC.

\section{Patients and methods}

Patient selection. A total of 325 patients with primary NPC were treated in The Department of Radiation Oncology of The Affiliated Hospital of Xuzhou Medical University between May 2008 and December 2016 and 52 patients were enrolled in the present study. The inclusion criteria were as follows: Pathologically confirmed NPC; $\geq 18$ years old; no previous abnormalities or surgical history involving the thyroid or the pituitary glands; treatment with radical IMRT; no serious complications of the liver, kidneys or heart; good compliance (periodic re-examination during the follow-up period); complete clinical information; and no evidence of distant metastasis or disease relapses. Exclusion criteria were as follows: Prior radiotherapy in the head and neck areas; evidence of malignant tumor types in other areas of the body; patients who received immunotherapy or hormonotherapy concurrently; and patients who are pregnant or lactating.

Based on the aforementioned criteria, 52 patients were eligible for the present study, including 30 males and 22 females. The median age of the patients was 50 years (age range, 18-75 years, mean 49.9 \pm 12.9 years). According to the American Joint Committee on Cancer staging system established in 2012 (8), 10 patients were in stage I-II, 24 in stage III, and 18 in stage IVA or IVB (Table I).

Radiotherapy. IMRT was delivered with Varian 23EX or UNIQUE medical linear accelerator, and $6 \mathrm{MV} X$ irradiation was administered. The targeted regions were divided into high-risk and low-risk regions. A low-risk region refers to a cervical lymphatic drainage area without metastasis. The radiation dose used was 1.8-2.0 Gy/fx28f, 5f/w, amounting to 50.4-56 Gy in total. A high-risk region refers to the entire nasopharynx, retropharyngeal lymph nodes, clivus, cranial base, parapharyngeal space, pterygopalatine fossa, sphenoid sinus, nasal cavity, the posterior third of the maxillary sinus and cervical lymphatic drainage areas with metastasis. The radiation dose used was 1.8-2.0 Gy/fx33f, 5f/w, amounting to 59.4-66 Gy in total. A simultaneously integrated boost was delivered to the primary tumor and positive lymph nodes were treated with a dose of 2.12-2.14 Gy/fx33f, 5f/w, and 69.96-70.62 Gy in total. Additionally, the radiation dose to the nasopharynx of patients with locally advanced NPC ranged from 71.3 to 74.9 Gy in total.

Measurement of thyroid function and the diagnostic criteria of hypothyroidism. Morning fasting plasma was collected to determine the levels of free triiodothyronine $\left(\mathrm{FT}_{3}\right)$, free tetraiodothyronine $\left(\mathrm{FT}_{4}\right)$ and thyroid stimulating hormone (TSH). The reference ranges were: $\mathrm{FT}_{3}, 2.8-7.1 \mathrm{pmol} / \mathrm{l}$; $\mathrm{FT}_{4}, 12-22 \mathrm{pmol} / \mathrm{l}$; and TSH, 0.27-4.2 mIU/1. TSH and $\mathrm{FT}_{4}$ were analyzed to distinguish central hypothyroidism from primary hypothyroidism. Additionally, high TSH levels in the presence of normal $\mathrm{FT}_{4}$ levels was referred to subclinical hypothyroidism, high TSH levels in the presence of low $\mathrm{FT}_{4}$ levels indicates the clinical subtype and low TSH levels is indicative of central hypothyroidism.

Dose-volume parameters of the thyroid and pituitary glands. The treatment plans for all patients were analyzed retrospectively. In 17 cases, different degrees of dose constraints were applied to the thyroid gland. Based on the computed topography (CT)-based simulation images obtained prior to and during radiotherapy, the thyroid and pituitary glands were delineated by a senior radiation oncologist who were blind to the study conditions. The relevant parameters, including mean dose (Dmean), maximum dose (Dmax), minimum dose and V5, V10, V20, V30, V40, V50, V60 and V70 (percentage of organ receiving at least 5, 10, 20, 30, 40, 50, 60 and $70 \mathrm{~Gy}$ ) were determined from dose-volume histograms.

Follow-up. Follow-up was initiated 3 months after the completion of radiotherapy. The frequency of follow-up visits was once every three months during the first 2 years after the completion of radiotherapy, and once every 6 months for the next 3 years, followed by once a year in the following period. The follow-up period was stopped when hypothyroidism occurred or no hypothyroidism occurred until the end of the follow-up. During the follow-up, peripheral $\mathrm{FT}_{3}, \mathrm{FT}_{4}$ and TSH levels were evaluated in addition to the regular magnetic resonance imaging/CT scan of the nasopharynx, chest CT scan, abdominal ultrasonography and routine blood test. The interval time of hypothyroidism was defined as the period between the end of radiotherapy and the initial occurrence of hematologic abnormality.

Statistical analysis. Data are presented as the mean \pm standard deviation. SPSS version 16.0 (SPSS, Inc., Chicago, IL, USA) was used for all statistical analyses. $\chi^{2}$ test was used to analyze enumeration data, while independent or paired Student's t-tests were applied for measurement data. The receiver operating characteristic (ROC) curves were used to determine the possible dose-volume threshold value. Kaplan-Meier survival curves were used to evaluate the cumulative incidence of RIHT and the log rank test was used to compare the survival curves. $\mathrm{P}<0.05$ (two-sided) was considered to indicate a statistically significant difference.

\section{Results}

Association between general clinical characteristics and RIHT. The median follow-up period was 17 months (range, 3-95 months). A total of 52 patients with NPC were divided into the euthyroid (24 patients) and hypothyroid groups (28 patients). In the hypothyroid group, 3 cases $(10.7 \%$ ) had central hypothyroidism, whereas the rest $(89.3 \%)$ had primary hypothyroidism (14 as clinical subtype and 11 as subclinical subtype). Additionally, 2 female patients with clinical hypothyroidism experienced a concurrent decrease in $\mathrm{FT}_{3}$ and $\mathrm{FT}_{4}$ levels, whereas the TSH levels (56 and >100 mIU/1, respectively) were notably elevated.

The incidence of RIHT was $72.7 \%(16 / 22)$ in female patients and $40 \%(12 / 30)$ in male patients, and there was a 
Table I. Clinical features of the enrolled patients.

\begin{tabular}{|c|c|c|}
\hline Clinical features & Number of cases & Percentage \\
\hline \multicolumn{3}{|l|}{ Sex } \\
\hline Male & 30 & 57.7 \\
\hline Female & 22 & 42.3 \\
\hline \multicolumn{3}{|l|}{ Age (years) } \\
\hline$<30$ or $>60$ & 11 & 21.2 \\
\hline $30-60$ & 41 & 78.8 \\
\hline \multicolumn{3}{|l|}{ Stage } \\
\hline I-II & 10 & 19.2 \\
\hline III-IV & 42 & 80.8 \\
\hline \multicolumn{3}{|l|}{ T stage } \\
\hline $\mathrm{T}_{1-2}$ & 24 & 46.2 \\
\hline $\mathrm{T}_{3-4}$ & 28 & 53.8 \\
\hline \multicolumn{3}{|l|}{$\mathrm{N}$ stage } \\
\hline $\mathrm{N}_{0-1}$ & 20 & 38.5 \\
\hline $\mathrm{N}_{2}$ & 23 & 44.2 \\
\hline $\mathrm{N}_{3}$ & 9 & 17.3 \\
\hline \multicolumn{3}{|l|}{$\begin{array}{l}\text { GTV simultaneous } \\
\text { integrated boost }\end{array}$} \\
\hline Yes & 45 & 86.5 \\
\hline No & 7 & 13.5 \\
\hline \multicolumn{3}{|l|}{ Dose constraint } \\
\hline Yes & 17 & 32.7 \\
\hline No & 35 & 67.3 \\
\hline
\end{tabular}

GTV, gross tumor volume.

significant different between them $(\mathrm{P}=0.010)$. The majority of female cases belonged to the clinical hypothyroidism subtype $(85.7 \%)$, while the majority of male patients belonged to the subclinical subtype $(81.8 \%)$. Although the difference in the median age between female and male patients was not statistically significant (48 years vs. 52 years; $\mathrm{P}=0.092$ ), the age distribution of the euthyroid and hypothyroid cohorts was distinct. A total of 9 patients in the hypothyroid group were $<30$ or $>60$ years $(32.1 \%)$, whereas only 2 patients in the euthyroid group (8.3\%) were these age groups, demonstrating a statistically significant difference $(\mathrm{P}=0.036)$; however, other characteristics, including clinical staging, $\mathrm{T}$ staging and $\mathrm{N}$ staging, were not significantly associated with RIHT (Table II).

Thyroid gland. The Dmean of euthyroid and hypothyroid

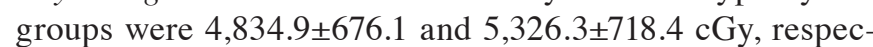
tively, and indicated significant differences $(\mathrm{P}=0.017)$. The disparity in V50 of the two groups (46.6 \pm 30.5 vs. $66.4 \pm 30.2 \%$, respectively) was also statistically significant $(\mathrm{P}=0.023)$, whereas no statistical significance was observed for Dmax between the two groups $(6,176.9 \pm 571.7$ vs. $6,490.5 \pm 569.4 \mathrm{cGy}$, respectively; $\mathrm{P}=0.060)$. Analysis of the ROC curves indicated that the threshold value was 5,160 cGy $(\mathrm{P}=0.024)$ for the Dmean and 54.5\% ( $\mathrm{P}=0.007)$ for the V50 (Fig. 1). Kaplan-Meier survival analysis demonstrated that the
Table II. The clinical characteristics of the euthyroid and hypothyroid groups.

\begin{tabular}{lccc}
\hline $\begin{array}{l}\text { Clinical } \\
\text { characteristics }\end{array}$ & $\begin{array}{c}\text { Euthyroid } \\
\text { group }\end{array}$ & $\begin{array}{c}\text { Hypothyroid } \\
\text { group }\end{array}$ & P-value \\
\hline Case number & 24 & 28 & \\
Sex & & 12 & $0.010^{\mathrm{a}}$ \\
Male & 18 & 16 & \\
Female & 6 & & \\
Age (years) & & 48 & 0.092 \\
Median & 52 & $18-75$ & $0.036^{\mathrm{a}}$ \\
Range & $35-69$ & 9 & 0.579 \\
$<30$ or $>60$ & 2 & 19 & \\
$30-60$ & 22 & & \\
Clinical stage & & 7 & 0.500 \\
Phase I-II & 3 & 21 & \\
Phase III-IV & 21 & 15 & \\
$\mathrm{~T}_{\text {stage }}$ & & 13 & \\
$\mathrm{~T}_{1-2}$ & 9 & 11 & \\
$\mathrm{~T}_{3-4}$ & 15 & & \\
$\mathrm{~N}_{\text {stage }}$ & & & \\
$\mathrm{N}_{0-1}$ & 9 & & \\
$\mathrm{~N}_{2}$ & 13 & & \\
$\mathrm{~N}_{3}$ & & & \\
\hline
\end{tabular}

${ }^{\mathrm{a}} \mathrm{P}<0.05$.

3-year cumulative incidence of RIHT was $67.8 \%$ when the Dmean was >5,160 cGy, which was significantly increased, compared with the cohort with Dmean $<5,160$ cGy (log rank test, $\mathrm{P}=0.036$ ). Furthermore, the 3 -year cumulative incidence of RIHT in patients with V50 $>54.5 \%$ was also significantly increased, compared with patients with V50 $<54.5 \%$ (66.1 vs. $29.9 \%$, respectively; log rank test, $\mathrm{P}=0.025$; Fig. 2). Regarding the thyroid volume, comparison of CT localization images indicated a significantly reduced thyroid volume $\left(15.8 \pm 6.8 \mathrm{~cm}^{3}\right.$ prior to treatment vs. $14.7 \pm 6.6 \mathrm{~cm}^{3}$ during treatment; $\mathrm{P}=0.002$ ). Furthermore, subgroup evaluation demonstrated that the thyroid volume in the euthyroid group was significantly increased, compared with the hypothyroid group $\left(17.85 \pm 5.89 \mathrm{~cm}^{3}\right.$ vs. $13.9 \pm 7.48 \mathrm{~cm}^{3}$, respectively; $\mathrm{P}=0.048)$. Of the 52 patients, $45(86.5 \%)$ patients had a simultaneously integrated boost of the gross tumor volume (GTV), but this treatment was not associated with the occurrence of RIHT ( $\mathrm{P}=0.670)$; however, dose constraints were applied in 17 patients $(32.7 \%)$, which significantly affected the incidence of RIHT ( $\mathrm{P}=0.034$; Table III).

Pituitary. The hypothalamus-pituitary-thyroid axis along with thyroid damage may impact the incidence of RIHT; therefore, in the present study, the pituitary-associated dose-volume parameters were analyzed. The present results demonstrated that the Dmean, V30, V40, V50 and V55 of the pituitary gland indicated no significant differences between the euthyroid and 

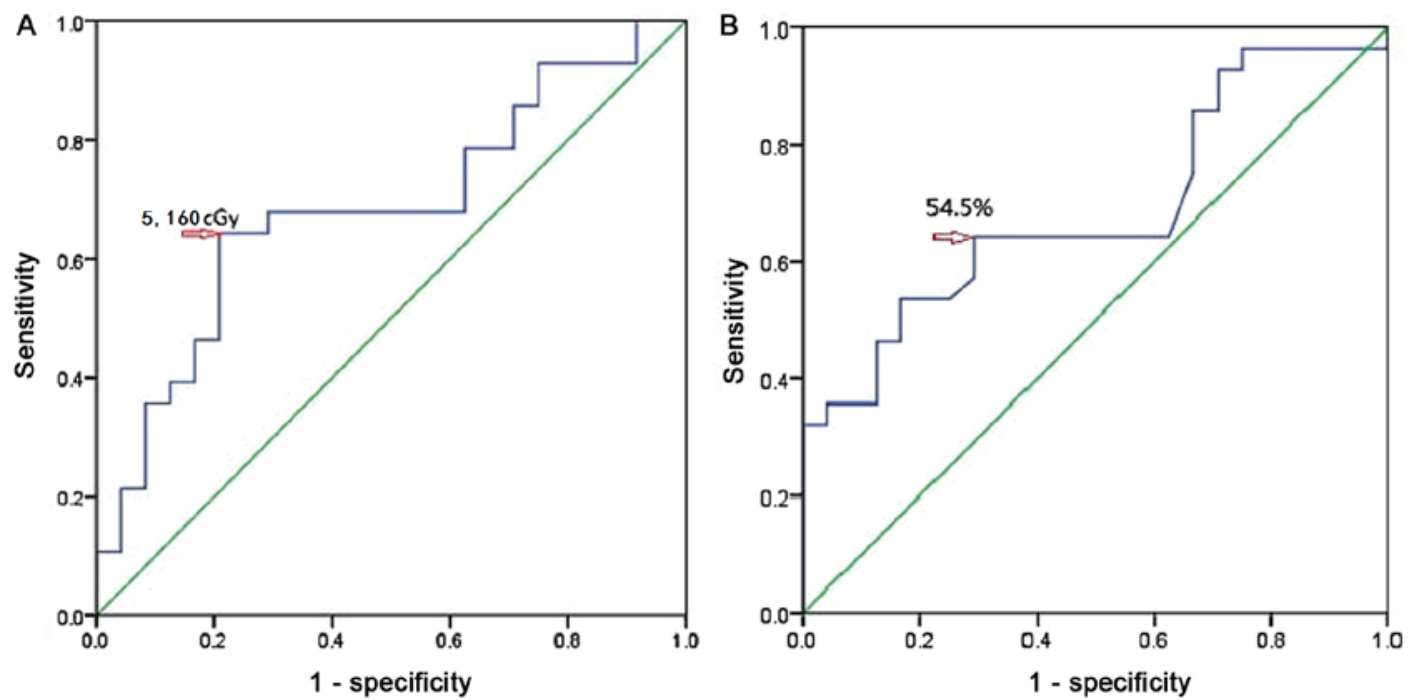

Figure 1. ROC curve analysis. ROC curves for (A) thyroid Dmean and (B) 50V. ROC, receiver operating characteristic; Dmean, mean dose.


Figure 2. Freedom from RIHT according to the thyroid-dose threshold. The cumulative incidence of RIHT was classified by two levels of (A) Dmean and (B) V50. Dmean, dose mean; RIHT, radiation-induced hypothyroidism. Freedom from RIHT, no RIHT.

hypothyroid groups $(\mathrm{P}>0.05)$; however, the exposure dose in the hypothyroid group was notably increased, compared with the euthyroid group (Table IV).

Cumulative incidence of RIHT. The incidence of RIHT was increased in a time-dependent manner post treatment. The results demonstrated cumulative incidence of $13,33,51$, 71 and $79 \%$, respectively, at 6 months, 1, 2,3 and 4 years (Fig. 3).

\section{Discussion}

The incidence of RIHT occurs in numerous cancer types, as demonstrated by a number of studies. Zohar et al (9) reported that thyroid dysfunction following radiotherapy occurred in $3 \%$ of patients with head and neck malignancy types, whereas Hancock et al (10) reported that the proportion of thyroid dysfunction was notable increased in the group who had received more than $30 \mathrm{~Gy}$ to the thyroid (44\%) compared with those who had not undergone irradiation $(2 \%)$ in patients with Hodgkin lymphoma. Recently, the radiation dose to the target region has been notably increased due to the usage of IMRT and intensity-modulated arc therapy (11); however, the key issue is the amount of radiation exposure to the organs at risk. RIHT is one of the most common complications observed in patients with head and neck neoplasms that underwent radiotherapy, which may occur due to insufficient dose constraint during the treatment as well as lack of follow-up care to ensure thorough thyroid function following treatment.

The median follow-up time to RIHT was 16.8-21.6 months from the end of radiotherapy (range, 3.6-86.4 months) $(12,13)$. The median follow-up time for the present study was 17 months (range, 3-95 months), and the incidence of RIHT was $53.8 \%$, and these results were consistent with the data of other previous studies $(12,13)$. In the present study, female patients were more susceptible to RIHT compared with males ( 72.7 vs. $40.0 \%$; $\mathrm{P}=0.010$ ), particularly for those in the clinical subtype (85.7\%). Furthermore, 2 female patients who exhibited clinical hypothyroidism experienced concurrent reduction of $\mathrm{FT}_{3}$ and $\mathrm{FT}_{4}$ levels accompanied by notably increased TSH levels, indicating severe thyroid dysfunction. The sex difference could be explained by the smaller volume of the female thyroid glands, compared with male thyroid 
Table III. Thyroid-associated dose-volume parameters of the euthyroid and hypothyroid groups.

\begin{tabular}{lccc}
\hline Variable & $\begin{array}{c}\text { Euthyroid } \\
\text { group }\end{array}$ & $\begin{array}{c}\text { Hypothyroid } \\
\text { group }\end{array}$ & P-value \\
\hline Dmean (cGy) & $4,834.9 \pm 676.1$ & $5,326.3 \pm 718.4$ & $0.017^{\mathrm{a}}$ \\
Dmax (cGy) & $6,176.9 \pm 571.7$ & $6,490.5 \pm 569.4$ & 0.060 \\
V50 (\%) & $46.6 \pm 30.5$ & $66.4 \pm 30.2$ & $0.023^{\mathrm{a}}$ \\
Original volume of & $17.9 \pm 5.9$ & $13.9 \pm 7.5$ & $0.048^{\mathrm{a}}$ \\
thyroid gland $\left(\mathrm{cm}^{3}\right)$ & & & \\
GTV boost & & & 0.670 \\
Yes & 20 & 3 & \\
No & 4 & & \\
Dose constraint & & 6 & \\
Yes & 11 & 22 & \\
No & 13 & & \\
\hline
\end{tabular}

${ }^{\mathrm{a}} \mathrm{P}<0.05$. GTV, gross tumor volume.

Table IV. Pituitary-associated dose-volume parameters of the euthyroid and hypothyroid groups.

\begin{tabular}{lccc}
\hline Variable & $\begin{array}{c}\text { Euthyroid } \\
\text { group }\end{array}$ & $\begin{array}{c}\text { Hypothyroid } \\
\text { group }\end{array}$ & P-value \\
\hline Dmean (cGy) & $4,117.9 \pm 1,779.5$ & $4,150.1 \pm 1,726.5$ & 0.949 \\
V30 (\%) & $75.7 \pm 37.9$ & $76.5 \pm 39.7$ & 0.949 \\
V40 (\%) & $57.4 \pm 40.4$ & $67.1 \pm 42.2$ & 0.417 \\
V50 (\%) & $34.4 \pm 40.1$ & $39.6 \pm 41.3$ & 0.650 \\
V55 (\%) & $21.5 \pm 34.9$ & $25.3 \pm 37.2$ & 0.717 \\
\hline
\end{tabular}

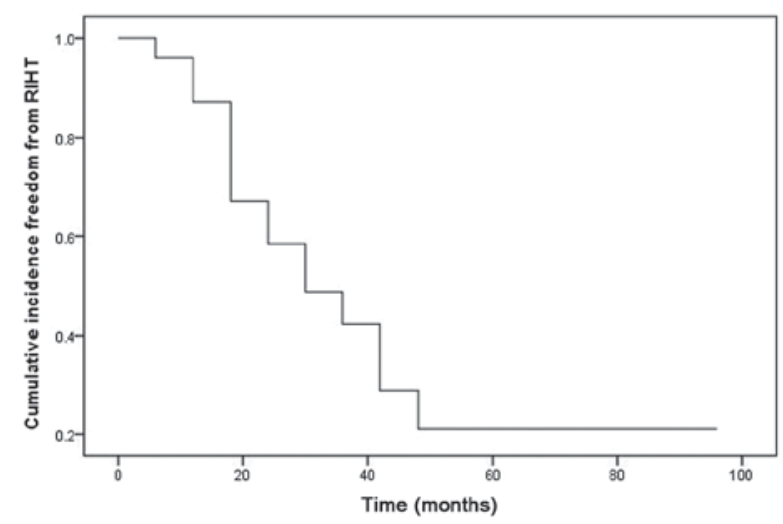

Figure 3. The cumulative incidence of RIHT. RIHT, radiation-induced hypothyroidism. Freedom from RIHT, no RIHT.

glands. Previous studies have demonstrated that thyroid gland size, particularly small thyroid glands, is a risk factor of RIHT $(14,15)$. Although the present study indicated no significant difference in the thyroid volume between males and females $\left(16.25 \pm 5.7\right.$ and $15.09 \pm 8.85 \mathrm{~cm}^{3}$, respectively; $\mathrm{P}=0.576)$, the thyroid volume prior to treatment was significantly associated with the incidence of RIHT $(\mathrm{P}=0.048)$, which demonstrated that patients with smaller thyroid glands have an increased probability to experience hypothyroidism; therefore, female patients who underwent radiation therapy may benefit from the extra measures to protect their thyroid gland from radiation exposure. Additionally, Lin et al (16) determined that thyroid volume was notably decreased within 6 months after radiotherapy. This reduction in thyroid gland size was also observed in the present study during treatment and indicated that thyroid damage may occur in the early stages of radiotherapy.

Age is an associated factor for the occurrence of hypothyroidism (17,18). Colevas et al (19) and Wu et al (20) reported that the age of the patients was associated with an increased risk for RIHT, particularly patients $<30$ and $>60$ years. Murthy et al (21) also determined that young people were more susceptible to hypothyroidism. Consistent with this, the present study results demonstrated that patients aged $>60$ and $<30$ years have an increased risk of hypothyroidism $(\mathrm{P}=0.036)$; therefore, dose limitations to the thyroid gland should be carefully applied in younger (aged $<30$ years) and older (aged $>60$ years) patients with NPC who receive radiotherapy.

$\mathrm{T}$ stage is another risk factor associated with hypothyroidism. Wu et al (20) determined that patients in stages $\mathrm{T}_{1-2}$ have a significantly increased probability to experience RIHT, compared with patients in stages $\mathrm{T}_{3-4}(\mathrm{P}=0.044)$. Furthermore, the statistical analysis of clinical stage, $\mathrm{T}$ stage, $\mathrm{N}$ stage and GTV boost did not determine any significant differences. In 52 patients, 10 cases were in stage I and II and RIHT occurred in seven of them. Retrospective analysis of the treatment plan for these 10 patients demonstrated that the lower bound of planning target volume (PTV) reached the cricothyroid membrane in 3 cases, and $\sim 2 \mathrm{~cm}$ above the sternoclavicular joint in the rest of the cases; therefore, a wide target region could contribute to the development of RIHT. In contrast, GTV boost demonstrated less impact on hypothyroidism, which may be explained by the distance between the thyroid gland and the boost area.

IMRT is considered as a primary radiotherapy approach and offers distinct dosimetric advantages, compared with conventional and three-dimensional conformal radiotherapy (3D-CRT). Diaz et al (18) demonstrated that dosing limitations to the thyroid gland during IMRT significantly reduced the Dmean, V30, V40 and V50 ( $\mathrm{P}<0.005)$. In the present study, dose constraint was conducted to various degrees, but was conducive in the prevention of hypothyroidism $(\mathrm{P}=0.034)$; therefore, dose constraint to the thyroid gland should be applied in patients with NPC who are subjected to radiotherapy.

Numerous studies have reported that RIHT is associated with the dose-volume parameters of the thyroid gland $(10,22,23)$; however, the nature of this association remains ambiguous. A Hodgkin lymphoma study by Cella et al (24) reported that V30 was an independent predictor for hypothyroidism. When V30 was $>62.5 \%$, the occurrence of hypothyroidism was significantly increased, compared with when V30 was $<62.5 \%$ (11.5\% vs. $70.8 \%$; $\mathrm{P}<0.0001)$. Similarly, studies on head and neck malignancy types conducted by Kim et al (25) and Sachdev et al (26) reported that V45 and V50 were independent predictors, the threshold values of which were 50 and $60 \%$, respectively. The present results demonstrated that the threshold value of V50 was $54.5 \%$ according to the ROC curve analysis. The 
3-year cumulative incidence for patients with V50 $>54.5 \%$ was $>2$-fold increased, compared with the patients with V50 $<54.5 \%$ (66.1\% vs. $29.9 \%$; $\mathrm{P}=0.025)$, which was consistent with the studies by Kim et al (25) and Sachdev et al (26); however, the threshold value may vary in different diseases and radiation doses. For instance, the prescribed dose for Hodgkin lymphoma ranged from 30-36 Gy, whereas the recommended dose for head and neck tumor types was 54-70 Gy; therefore, the volume parameters reported by Cella et al (24) was notably reduced. The Dmean has also been reported as an independent predictor for RIHT $(27,28)$; however, the associated threshold value has rarely been studied. Fujiwara et al (29) estimated the threshold value as $30 \mathrm{~Gy}$, based on the incidence of RIHT being significantly reduced in patients with Dmean $<30$ Gy compared with the other groups $(\mathrm{P}<0.05)$. The present ROC curve analysis demonstrated that 5,160 cGy was a predictive threshold value, and the increased 3-year cumulative incidence of RIHT in the Dmean $>5,160$ cGy cohort confirmed this data $(67.8 \%$ for Dmean $>5,160 \mathrm{cGy}$ and $44.6 \%$ for Dmean $<5,160 \mathrm{cGy}$; $\mathrm{P}=0.036)$. Notably, the threshold value in the present study was $>30$ Gy reported by Fujiwara et al (29). Possible explanations are as follows: i) The dose in the target region was 54-60 Gy, and the prescribed dose was 59.4-66 Gy for PTV and 70-74.9 Gy for GTV boost, whereas the total dose in the Fujiwara et al (29) study was 60-66 Gy; and ii) The difference in the tumor types (NPC vs. head and neck malignancy types) and physicians may result in variations in the target area delineations. In the present study, $30.6 \%$ (range, 1-88.2\%) of thyroid tissues were exposed to radiation, resulting in the exposure of increased radiation doses to the thyroid gland. In clinical practice, the threshold value not only can be applied as a reference to evaluate the risk of RIHT, but could also serve as an indicator to take preventive care measures in high-risk patients as early as possible.

The thyroid gland is more susceptible to secondary injuries during radiotherapy in patients with NPC due to a large target area, including the proximity of the hypothalamus and pituitary gland. Huang et al (30) demonstrated that the increase of Dmean $(\mathrm{P}=0.009)$ and V55 $(\mathrm{P}=0.014)$ of the pituitary gland was significantly associated with the increased TSH levels. The present study did not determine an association between the pituitary radiation dose and RIHT; however, the Dmean, V30, V40, V50 and V55 of the pituitary gland were increased in the hypothyroid group, compared with the euthyroid group. This may be due to the thyroid and pituitary gland concurrently influencing the thyroid function, and hence it is necessary to limit the radiation dose to the pituitary gland.

To conclude, the present study indicated that the incidence of RIHT in patients with NPC was associated with sex and age, as well as the Dmean and V50 of the thyroid gland. The original gland volume prior to radiotherapy and dose constraints associated with the gland demonstrated significant impact on the incidence of RIHT; however, due to the lack of standardization of dose constraints and a small cohort size, subgroup analysis was not performed and the optimal radiation dose was not determined. Future prospective studies should investigate threshold values with increase accuracy to reduce RIHT-associated morbidity and to improve the QoL of patients.

\section{Acknowledgements}

Not applicable.

\section{Funding}

The Project of Invigorating Health Care through Science, Technology and Education Jiangsu Provincial Medical Innovation Team, The Project of Science and Technology of Jiangsu Provincial Commission Health and Family planning (grant no. H201426), Xuzhou City Science and Technology Bureau issues (grant nos. KC15SH024 and KC16SH065) and The Project or Invigorating Health Care through Science, Technology and Education (grant no. CXTDA2017034).

\section{Availability of data and materials}

The datasets analyzed for the current study are available from the corresponding author upon request.

\section{Authors' contributions}

LZ and JW conceived the study. YX and ZS designed the study. YX, TT, GL and YY made substantial contributions towards the acquisition of data. YX conducted the statistical analysis. YX and ZS drafted the manuscript. ZS, LZ and JW critically revised the manuscript. All authors reviewed and approved the final paper.

\section{Ethics approval and consent to participate}

This is a retrospective analysis of clinical data without treatment intervention and personal identification. Therefore, formal consent from our ethics committee is not necessary.

\section{Patient consent for publication}

Not applicable.

\section{Competing interests}

The authors declare that they have no competing interests.

\section{References}

1. Yi HM, Yi H, Zhu JF, Xiao T, Lu SS, Guan YJ and Xiao ZQ: A five-variable signature predicts radioresistance and prognosis in nasopharyngeal carcinoma patients receiving radical radiotherapy. Tumour Biol 37: 2941-2949, 2016.

2. Boomsma MJ, Bijl HP and Langendijk JA: Radiation-induced hypothyroidism in head and neck cancer patients: A systematic review. Radiother Oncol 99: 1-5, 2011.

3. Vanderpump MP and Tunbridge WM: Epidemiology and prevention of clinical and subclinical hypothyroidism. Thyroid 12: 839-847, 2002.

4. Edge SB, Byrd DR, Compton CC, Fritz AG, Greene FL and Trotti A (eds): AJCC cancer staging manual. 7th edition. Springer-Verlag, New York, NY, 2010.

5. Tian YM, Xiao WW, Bai L, Liu XW, Zhao C, Lu TX and Han F: Impact of primary tumor volume and location on the prognosis of patients with locally recurrent nasopharyngeal carcinoma. Chin J Cancer 34: 247-253, 2015.

6. Duntas LH and Brenta G: The effect of thyroid disorders on lipid levels and metabolism. Med Clin North Am 96: 269-281, 2012. 
7. Laulund AS, Nybo M, Brix TH, Abrahamsen B, Jørgensen HL and Hegedüs L: Duration of thyroid dysfunction correlates with all-cause mortality. The OPENTHYRO register cohort. PLoS One 9: e110437, 2014.

8. Amin MB, Edge S, Greene F, Byrd DR, Brookland RK, Washington MK, Gershenwald JE, Compton CC, Hess KR, Sullivan DC, et al (eds): AJCC Cancer Staging Manual. Eigth edition. Springer-Verlag, New York, NY, 2017.

9. Zohar Y, Tovim RB, Laurian N and Laurian L: Thyroid function following radiation and surgical therapy in head and neck malignancy. Head Neck Surg 6: 948-952, 1984.

10. Hancock SL, Cox RS and McDougall IR: Thyroid disease after treatment of Hodgkin'S disease. N Engl J Med 325: 599-605, 1991.

11. Peng YL, Chen L, Shen GZ, Li YN, Yao JJ, Xiao WW, Yang L, Zhou S, Li JX, Cheng WQ, et al: Interobserver variations in the delineation of target volumes and organs at risk and their impact on dose distribution in intensity-modulated radiation therapy for nasopharyngeal carcinoma. Oral Oncol 82: 1-7, 2018.

12. Mercado G, Adelstein DJ, Saxton JP, Secic M, Larto MA and Lavertu P: Hypothyroidism: A frequent event after radiotherapy and after radiotherapy with chemotherapy for patients with head and neck carcinoma. Cancer 92: 2892-2897, 2001.

13. Tell R, Lundell G, Nilsson B, Sjödin H, Lewin F and Lewensohn R: Long-term incidence of hypothyroidism after radiotherapy in patients with head-and-neck cancer. Int J Radiat Oncol Biol Phys 60: 395-400, 2004.

14. Cella L, Liuzzi R, Conson M, D'Avino V, Salvatore M and Pacelli R: Development of multivariate NTCP models for radiation-induced hypothyroidism: A comparative analysis. Radiat Oncol 7: 224, 2012.

15. Feen Rønjom M: Radiation-induced hypothyroidism after treatment of head and neck cancer. Dan Med J 63: 2016.

16. Lin Z, Wu VW, Lin J, Feng H and Chen L: A longitudinal study on the radiation-induced thyroid gland changes after external beam radiotherapy of nasopharyngeal carcinoma. Thyroid 21 19-23, 2011.

17. Tell R, Sjödin H, Lundell G, Lewin F and Lewensohn R: Hypothyroidism after external radiotherapy for head and neck cancer. Int J Radiat Oncol Biol Phys 39: 303-308, 1997.

18. Diaz R, Jaboin JJ, Morales-Paliza M, Koehler E, Phillips JG, Stinson S, Gilbert J, Chung CH, Murphy BA, Yarbrough WG, et al: Hypothyroidism as a consequence of intensity-modulated radiotherapy with concurrent taxane-based chemotherapy for locally advanced head-and-neck cancer. Int J Radiat Oncol Biol Phys 77 : 468-476, 2010

19. Colevas AD, Read R, Thornhill J, Adak S, Tishler R, Busse P, Li Y and Posner M: Hypothyroidism incidence after multimodality treatment for stage III and IV squamous cell carcinomas of the head and neck. Int J Radiat Oncol Biol Phys 51: 599-604 2001.
20. Wu YH, Wang HM, Chen HH, Lin CY, Chen EY, Fan KH, Huang SF, Chen IH, Liao CT, Cheng AJ and Chang JT: Hypothyroidism after radiotherapy for nasopharyngeal cancer patients. Int J Radiat Oncol Biol Phys 76: 1133-1139, 2010.

21. Murthy V, Narang K, Ghosh-Laskar S, Gupta T, Budrukkar A and Agrawal JP: Hypothyroidism after 3-dimensional conformal radiotherapy and intensity-modulated radiotherapy for head and neck cancers: Prospective data from 2 randomized controlled trials. Head Neck 36: 1573-1580, 2014

22. August M, Wang J, Plante D and Wang CC: Complications associated with therapeutic neck radiation. J Oral Maxillofac Surg 54: 1409-1416, 1996.

23. Grande C: Hypothyroidism following radiotherapy for head and neck cancer: Multivariate analysis of risk factors. Radiother Oncol 25: 31-36, 1992

24. Cella L, Conson M, Caterino M, De Rosa N, Liuzzi R, Picardi M, Grimaldi F, Solla R, Farella A, Salvatore M and Pacelli R: Thyroid V30 predicts radiation-induced hypothyroidism in patients treated with sequential chemo-radiotherapy for Hodgkin's lymphoma. Int J Radiat Oncol Biol Phys 82: 1802-1808, 2012.

25. Kim MY, Yu T and Wu HG: Dose-volumetric parameters for predicting hypothyroidism after radiotherapy for head and neck cancer. Jpn J Clin Oncol 44: 331-337, 2014.

26. Sachdev S, Refaat T, Bacchus ID, Sathiaseelan V and Mittal BB: Thyroid V50 highly predictive of hypothyroidism in head-and-neck cancer patients treated with intensity-modulated radiotherapy (IMRT). Am J Clin Oncol 40: 413-417, 2017.

27. Boomsma MJ, Bijl HP, Christianen ME, Beetz I, Chouvalova O, Steenbakkers RJ, van der Laan BF, Wolffenbuttel BH, Oosting SF, Schilstra C and Langendijk JA: A prospective cohort study on radiation-induced hypothyroidism: Development of an NTCP model. Int J Radiat Oncol Biol Phys 84: e351-e363, 2012.

28. Akgun Z, Atasoy BM, Ozen Z, Yavuz D, Gulluoglu B, Sengoz M and Abacioglu U: V30 as a predictor for radiation-induced hypothyroidism: A dosimetric analysis in patients who received radiotherapy to the neck. Radiat Oncol 9: 104, 2014.

29. Fujiwara M, Kamikonya N, Odawara S, Suzuki H, Niwa Y, Takada Y, Doi H, Terada T, Uwa N, Sagawa K and Hirota S: The threshold of hypothyroidism after radiation therapy for head and neck cancer: A retrospective analysis of 116 cases. J Radiat Res 56: 577-582, 2015.

30. Huang S, Wang X,HuCand Ying H:Hypothalamic-pituitary-thyroid dysfunction induced by intensity-modulated radiotherapy (IMRT) for adult patients with nasopharyngeal carcinoma. Med Oncol 30: 710, 2013. 